\title{
IL-2 and zoledronic acid therapy restores the in vivo anti-leukemic activity of human lymphocytes pre- exposed to simulated microgravity
}

Preteesh Leo Mylabathula

University of Arizona

Douglass M. Diak

University of Arizona

Forrest L. Baker

University of Arizona

Grace M. Niemiro

University of Arizona

Melissa M. Markofski

University of Houston

Brian E. Crucian

Johnson Space Center

Emmanuel Katsanis

University of Arizona

Richard J. Simpson ( $\nabla$ rjsimpson@email.arizona.edu )

University of Arizona

\section{Research Article}

Keywords: Spaceflight, immunology, rotary cell culture system, natural killer cells, humanized mice, cancer

Posted Date: February 28th, 2022

DOI: https://doi.org/10.21203/rs.3.rs-1374348/v1

License: (c) (i) This work is licensed under a Creative Commons Attribution 4.0 International License.

Read Full License 


\section{Abstract}

Background: We have previously shown that the anti-tumor activity of human lymphocytes is diminished in vitro after 12-hours pre-exposure to simulated microgravity (SMG). Here we used an immunocompromised mouse model to determine if this loss of function would extend in vivo, and to also test the efficacy of IL-2 and zoledronic acid (ZOL) therapy as a potential countermeasure against SMGinduced immune dysfunction. We adoptively transferred human lymphocytes that were exposed to either SMG or 1G-control into NSG-Tg(Hu-IL 15) mice 1-week after they were injected with a luciferase-tagged human chronic myeloid leukemia (K562) cell line. Tumor growth was monitored 2x weekly with bioluminescence imaging (BLI) for up to 6-weeks.

Results: Mice that received lymphocytes exposed to SMG showed greater tumor burden compared to those receiving lymphocytes exposed to $1 \mathrm{G}$ (week 6 BLI: $1.8 \mathrm{e}^{10} \pm 8.07 \mathrm{e}^{9}$ versus $2.22 \mathrm{e}^{8} \pm 1.39 \mathrm{e}^{8}$ photons/second; $\mathrm{p}<0.0001)$. Peak BLI was also higher in the SMG group compared to $1 \mathrm{G}$-control $\left(2.34 \mathrm{e}^{10}\right.$ $\pm 1.23 \mathrm{e}^{10}$ versus $3.75 \mathrm{e}^{8} \pm 1.56 \mathrm{e}^{8}$ photons/second; $p=0.0062$ ). Exposure to SMG did not affect the ability of human lymphocytes to engraft or evoke xeno-graft-versus-host disease in the mice. Additionally, we injected the mice with IL-2 and zoledronic acid (ZOL) to expand and activate the anti-tumor activity of NK cells and $y \delta-T$ cells, respectively. This was found to revive the loss in anti-leukemic function observed in vivo when lymphocytes were pre-exposed to SMG.

Conclusion: Microgravity plays a contributory role in loss of tumor control in vivo. Immuno-stimulating agents like ZOL+IL-2 may offer an important countermeasure for immune dysregulation during prolonged spaceflight.

\section{Background}

Immune dysregulation has been reported in astronauts following both short (14-17 days $)^{1-4}$ and long (5-6 months) $)^{5,6}$ duration spaceflight. This includes diminished cell mediated immunity with a Th-2 cytokine shift, with latent viral reactivation being a frequent manifestation of immune dysregulation in astronauts. Many factors including microgravity, space radiation, isolation and confinement stress, altered circadian rhythms, nutritional deficiencies, and the gut microbiome are believed to play a role in altering astronaut immunity during extended space voyages ${ }^{7}$.

A particular concern for astronauts is that space travel might impair anti-tumor immune surveillance, increasing cancer risk in future exploration class mission crew. We showed previously that the anti-tumor activity of natural killer (NK) cells was diminished in crewmembers onboard the International Space Station ${ }^{8}$. We further demonstrated that pre-exposing human lymphocytes to $12 \mathrm{~h}$ of simulated microgravity (SMG), impaired NK cell killing against a range of hematological cancer cell lines in vitro when returned to the $1 \mathrm{G}$ environment ${ }^{9}$. The SMG exposed NK cells expressed lower levels of the cytolytic granules perforin and granzyme b, and were less capable of degranulation (CD107a+) and secreting effector cytokines (e.g. TNF-a and IFN- $y$ ) when co-cultured with tumor target cells after being pre-exposed 
to SMG. Exposing K562 chronic myeloid leukemia (CML) cells to SMG did not alter their susceptibility to NK cell killing, indicating that cancer cells might be more resistant to the effects of SMG compared to primary immune cells. This might tip the balance in the favor of tumor transformation during a long duration mission, particularly against a backdrop of continuous radiation exposure and further immune suppression due to latent viral reactivation.

A limitation of our previous work was the reliance on in vitro assays to assess the anti-tumor activity of human lymphocytes collected from astronauts in space or following exposure to $\mathrm{SMG}^{8,9}$. Unfortunately, these assays fail to capture other key elements of the anti-tumor response unique to in vivo setting including extravasation, homing, tumor infiltration, and killing. Humanized mouse models offer an excellent platform to determine the anti-tumor activity of human immune cells in vivo. NSG-Tg(Hu-IL15) animals harbor Prkdc ${ }^{\text {scid } 10}$ and IL2rg ${ }^{\text {tm1 } 1 \text { Wj } 11-13}$ mutations and an insertion of humanized IL-15 sequence $(\mathrm{Tg}(\mathrm{IL} 15) 1 \mathrm{Sz} / \mathrm{SzJ})$ that allows improved engraftment of primary human cells ${ }^{11}$ and increased survival of NK cells ${ }^{14}$. This mouse model also allows us to test the effectiveness of certain pharmacological countermeasures that have been proposed to mitigate spaceflight associated immune dysregulation ${ }^{15}$.

Administration of subcutaneous interleukin-2 (IL-2) has shown to improve anti-tumor activity of NK cells in pre-clinical mouse models ${ }^{16,17}$ and in various clinical trials ${ }^{18-20}$. Zoledronic acid is an aminobisphosphonate with clinical therapeutic applications including improving bone mineral density 21,22 and anti-tumor immunity through suppression of $\mathrm{T}_{\text {regs }}{ }^{23,24}$. Zoledronic acid has also been shown to expand

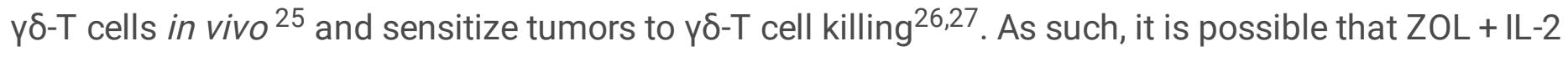
could restore the anti-leukemic activity of human effector lymphocytes pre-exposed to SMG.

The aim of this study was to determine if pre-exposure to SMG would impair the anti-leukemic function of human lymphocytes in vivo using the NSG-Tg(Hu-IL15) mouse model (Fig. 1). K562 leukemia bearing mice were engrafted with human immune cells exposed to either SMG or $1 \mathrm{G}$ and monitored for tumor growth over 6-weeks via bioluminescent imaging. Additionally, we tested if ZOL + IL-2 administered in vivo could revive SMG-induced loss of anti-leukemic function.

\section{Methods}

\section{Participants and blood collection}

Healthy adults between the age of 18-49 years volunteered for the study. Each participant provided written informed consent and had a single blood sample drawn by standard phlebotomy. Blood was collected in acid citrate dextrose (ACD) tubes and peripheral blood mononuclear cells (PBMCs) were isolated using density gradient separation method with Ficoll (Sigma-Aldrich, St. Louis, MO, USA) and processed immediately. All study procedures were approved by the Institutional Review Board (IRB) at University of Arizona. 


\section{Rotary cell culture system}

We exposed human PBMCs to simulated microgravity (SMG) or 1-G control as previously described ${ }^{9}$. Briefly, a rotary cell culture system (RCCS) (Synthecon, Houston, TX, USA) was used to simulate microgravity. PBMCs were resuspended in 10\%FBS+RPMI media in $10 \mathrm{ml}$ high-aspect ratio vessels (HARVs) and exposed to simulated microgravity for 12-hours while rotating on a horizontal axis at 10RPM. A 1G-control HARV was rotated on a vertical axis exposing PBMCs to similar shear stress while experiencing $1 \mathrm{G}$ gravitational force.

\section{NSG-Tg(Hu-IL15) mouse model}

All mouse experiments were done in compliance with Institutional Animal Care and Use Committee (IACUC) guidelines at the University of Arizona under an approved protocol. NSG-Tg(Hu-IL15) animals were purchased from Jackson laboratory (Bar Harbor, ME, USA) and breeding colonies were maintained at the University Animal Care facility. Animals were housed for the duration of the experiments at the University Animal Care facility.

Animals were irradiated with $100 \mathrm{cGy}$ in a Cesium ${ }^{137}$ irradiator (Atomic Energy of Canada Limited, Chalk River, Canada) a day before they were injected with 1 million luciferase-tagged-k562 (chronic myeloid leukemia) cells (figure 1). One week later, they were injected with 10 million PBMCs from SMG or $1 \mathrm{G}$ control conditions. 'Tumor only' control group received only 1 million K562 cells with placebo (PBS) on the day of immune cells injection. Bioluminescent intensity, weight, morbidity, and GVHD were monitored twice a week. Engraftment data was also collected from another group of mice that received only immune cells without tumor cells to examine differences in engraftment in the absence of tumor. Blood draw was performed from the sub-mandibular vein once a week to examine engraftment dynamics. Animals were sacrificed when they reached sacrificial criteria or at the end of the experiment (6 weeks). Since we propose to study the effect SMG on in vivo anti-leukemic activity of human PBMCs, which is largely driven by NK cells, we used this NSG-Tg(Hu-IL15) mouse model, which enhances in vivo survival of human NK cells. Our experiments suggested that acute GVHD signs occur around 14 days after PBMC injection and severe GVHD occurs around day 28. Therefore, we chose to evaluate graft-versus-tumor effect before severe GVHD starts setting in to prevent GVHD associated immune modulation from eclipsing the graft-versus-tumor effect of human PBMCs. Acute GVHD scoring was based on 6 parameters in accordance with previously published grading criteria ${ }^{28,29}$. All animals were terminated if they presented with a GvHD score of 4 or more or had lost more than $20 \%$ of their initial body weight.

Administration of zoledronic acid and interleukin-2: Subgroups of mice received an intraperitoneal injection of zoledronic acid $(50 \mu \mathrm{g} / \mathrm{kg})$ with a subcutaneous injection of IL-2 $(40,000 \mathrm{I.U}) 1 \mathrm{x}$ weekly starting at day 7 (i.e. the day of PBMC transfer) of the experiment (fig.1). 


\section{Bioluminescent imaging}

K-562-luc2 (ATCC $\AA$ CCL-243-LUC2 ${ }^{\text {TM }}$ ) (American Type Culture Collection, Manassas, VA, USA), which is a K562 chronic myeloid leukemia cell line that has been transfected with luciferase, was used for the in vivo experiments ${ }^{30,31}$. Tumor burden was measured immediately after D-luciferin (GoldBio, St Louis, MO) was injected intraperitoneally. A LagoX spectral imager (Spectral Instruments Imaging, Tucson, AZ) was used to quantify bioluminescent intensity (BLI) scores.

\section{Flow cytometry}

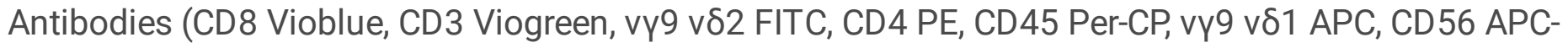
Vio770, CD16 Vioblue, NKG2D PE, PD-1 Per-CP, CD158b PE-Vio770, NKG2A APC, TCR-үठ APC-Vio770, NKp30 PE) (Miltenyi Biotec, San Diego, CA) were added to $25 \mu \mathrm{L}$ of whole blood at the proper titrated volumes and allowed to incubate for 30 minutes at room temperature in the dark. Blood samples were lysed with 1X Red Blood Cell Lysis solution (Miltenyi Biotec, San Diego, CA), washed twice in phosphate-

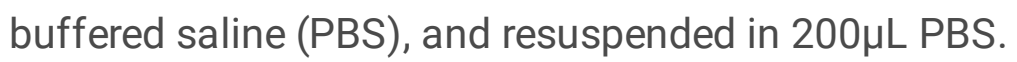

\section{Statistical analysis}

Statistical analysis was performed on Graphpad Prism 8.4.3 software (Graphpad, San Diego, CA). For tumor progression, bioluminescent intensity data was log-transformed for normality as previously indicated ${ }^{32}$. A mixed effects model was used to analyze differences between tumor growth with either 3 (e.g. SMG, 1G, vehicle) or 5 (e.g. SMG, SMG+ZOL+IL-2, 1G, vehicle, vehicle+ZOL+IL-2) 'condition' levels included in the model, along with a main effect for 'time' and 'condition*time' included as an interaction effect. When the interaction effect was significant (indicating that tumor growth differed between conditions as the experiment progressed), systematic pairwise comparisons were conducted with Bonferroni correction to identify which conditions were significantly different from each other over time. Peak BLI was analyzed using Friedman test for paired comparisons. GVHD scores and engraftment data were analyzed using a linear mixed model or a two-way RM ANOVA. A log-rank (Mantel-Cox) test was used for survival analysis. Statistical significance was set at $p<0.05$.

\section{Results}

Exposure to SMG impaired the anti-leukemic activity of human immune cells in vivo.

To evaluate the effect of SMG on anti-leukemia activity of human immune cells in vivo we compared tumor growth between mice that were injected with PBMCs exposed to SMG (TUMOR + SMG PBMCs) or 1G-control (TUMOR + 1G PBMCs). TUMOR + vehicle (PBS) was used as a control reference for unrestrained tumor growth. Representative BLI images showing tumor dynamics in vivo are shown in Fig. 2. For tumor growth, statistically significant effects were found for time, condition, and 
time*condition $(p<0.0001)$ (Fig. 3a). Post-hoc analyses revealed these effects to be driven by a lower tumor burden in the mice transferred with $1 \mathrm{G}$ exposed PBMCs compared to mice transferred with SMG exposed PBMCs and vehicle. Tumor growth kinetics were similar between SMG exposed PBMCs and vehicle control $(p>0.05)$. Peak tumor growth (as determined by highest BLI score) was significantly higher in the mice receiving SMG exposed PBMCs and vehicle compared to mice receiving $1 \mathrm{G}$ exposed PBMCs. There were no significant differences for peak tumor growth between the SMG exposed PBMC and vehicle conditions $(p>0.05)$. Overall survival up to 6 -weeks did not differ significantly across the conditions $(X 2=2.934, p=0.231)$ (Fig. $3 c)$. For GvHD/morbidity score, an expected significant effect was seen for time $(p<0.0001)$, but not 'condition' $(p=0.764)$ or 'condition*time' $(p=0.981)$ (Fig. $3 d)$.

\section{Exposure to SMG did not affect human immune cell engraftment in vivo.}

To verify that exposure to SMG did not impair the PBMCs' ability to engraft and expand in a xenograft; mice were injected with PBMCs only and not tumor. Engraftment was measured as a proportion of CD45human+ $(C D 45 h+)$ cells in total immune cells in mice blood collected at weekly intervals from week 2-5. The engraftment dynamics of PBMCs in the mice were not statistically different between SMG and $1 \mathrm{G}$ conditions (Fig. 4). This was true for total PBMCs as defined by human CD 45 expression as a percentage of the total human + mouse CD45 expressing cells (\% $p=0.175$; cells $/ \mathrm{uL}, p=0.628$, Fig. $4 a$, 4c.) and for NK-cells (CD3-/CD56+) expressed as a percentage of CD45h + cells (\% $p=0.219 ; \mathrm{cells} / \mu \mathrm{l}, \mathrm{p}=$ 0.478 , Fig. 4b, 4d.). Similar human immune cell engraftment dynamics were observed in mice with tumor (Fig. 5).

\section{Zoledronic acid and IL-2 therapy improved anti-leukemia activity of human immune cells in vivo.}

In an attempt to 'rescue' the anti-leukemic effects of PBMCs pre-exposed to SMG prior to adoptive transfer, we injected mice with ZOL + IL-2 on the day of PBMC transfer and once weekly thereafter. For tumor growth, statistically significant effects were found for time, condition, and time*condition $(p<$ 0.0001) (Fig. 6a). As observed in the previous experiment, 1G exposed PBMCs significantly inhibited tumor growth compared to SMG exposed PBMCs. Post-hoc analysis revealed a significant interaction effect $(p=0.0004)$ between TUMOR + SMG PBMCs and TUMOR + SMG PBMCs + ZOL + IL2, while no interaction effects were found for TUMOR + SMG PBMCs + ZOL + IL2 versus TUMOR + 1G PBMCs $(p=$ 0.567). There were also no significant differences for condition $(p=0.947)$ or time $(p=0.221)$ between these groups. This revealed that ZOL + IL2 administration enabled SMG-exposed PBMCs to control tumor growth rate as efficiently as $1 \mathrm{G}$-exposed PBMCs. As expected, ZOL + IL-2 had no effect on tumor growth dynamics in the absence of PBMCs $(p>0.05)$. Peak tumor growth (as determined by highest BLI score) was significantly higher in the mice receiving SMG exposed PBMCs and vehicle (with or without ZOL + IL2) compared to mice receiving $1 G$ exposed PBMCs and SMG exposed PBMCs + ZOL + IL-2. There were no significant differences for peak tumor growth between the $1 \mathrm{G}$ exposed PBMCs and the SMG exposed PBMCs + ZOL + IL-2 ( $>$ > 0.05), again indicating that the administration of ZOL + IL-2 rescued the antileukemic effect of PBMCs pre-exposed to SMG. Overall survival up to 6-weeks did not differ significantly 
across the conditions $(X 2=2.429, p=0.6575)$ (Fig. $6 c)$. Administration of $Z O L+I L 2$ therapy did not affect GVHD/morbidity progression ( $p>0.999)$ (Fig. 6d).

\section{Zoledronic acid and IL-2 therapy did not alter the number of NK and $\gamma \delta-T$ cells in blood.}

To determine if ZOL + IL2 therapy increased the number and proportion of NK cells and $y \delta-T$ cells in peripheral blood, weekly human leukocyte engraftment checks were performed after adoptive transfer (Fig. 7). No significant interaction*time effects were found for total CD45h+ $(\% p=0.713$; cells $/ u L, p=$ 0.419, Fig. 7a, 7b), NK cells (\% $p=0.99$; cells $/ u L, p=0.941$, Fig. 7c, 7d), or $\gamma \delta-T$ cells $(\% p=0.915$; cells $/ u L$, $p=0.40 .937$, Fig. 7e, 7f), indicating that ZOL + IL-2, despite increasing tumor control, did not expand these leukocyte populations in peripheral blood.

\section{Discussion}

The overarching aims of this study were to determine if the loss of NK-cell function against a leukemic cell line in vitro due to exposure to simulated microgravity (SMG) would extend to an in vivo model, and to also test the efficacy of zoledronic acid and IL-2 (ZOL + IL-2) therapy as a countermeasure to SMGinduced immune dysfunction. Using a human K562 tumor bearing immunocompromised mouse model, we found that pre-exposing human PBMCs to SMG for $12 \mathrm{~h}$ inhibits their ability to control leukemic growth in vivo after adoptive transfer, but systemic administration of ZOL + IL-2 'rescued' this SMG induced impairment of anti-leukemic activity. These findings indicate that microgravity likely plays a contributory role to immune system impairment during long duration space travel. Further, ZOL + IL-2 (a treatment that is currently used to expand and increase the anti-leukemic activity of NK-cells and $\gamma \delta-T$ cells in cancer patients ${ }^{19,20,33}$ ) could serve as an effective countermeasure to mitigate deficits in antitumor immunity due to prolonged exposure to microgravity during extended space missions.

This is the first study, to our knowledge, to show that pre-exposing primary human lymphocytes to SMG inhibits their ability to control leukemic growth in vivo. Not only were the SMG exposed PBMCs less effective at controlling leukemic growth in comparison to their $1 \mathrm{G}$ exposed counterparts, tumor growth kinetics were identical between the SMG exposed and vehicle control conditions indicating that SMG completely incapacitates the anti-leukemic activity of human lymphocytes. This corroborates our previous findings that pre-exposing human lymphocytes to SMG impairs their ability to kill various hematologic cancer cells lines in vitro ${ }^{34}$. While the mechanisms underpinning this response are not fully known, our prior in vitro studies showed that SMG impairs several anti-tumor properties of NK-cells, including their ability to deliver 'lethal hits' through cytotoxic degranulation and secretion of effector cytokines $^{34}$. Although it is possible that SMG could have impaired human cell engraftment in the mouse, we somewhat alleviated this potential confounder by showing similar levels of human leukocyte engraftment between the SMG and $1 G$ conditions. While blood levels of immune cells might not be reflective of engraftment levels in tissues or in mice with tumor, this showed that human immune cells (including NK cells) thrived similarly in mice after exposure to $1 \mathrm{G}$ and SMG. In vivo expansion of (CD45h + CD3-CD56+) human NK cells in the initial weeks was to be expected in an NSG-tg(hu-IL15) mouse 
model. NSG-tg(hu-IL15) mice sustain IL-15 blood levels of $7.1+/-0.3 \mathrm{pg} / \mathrm{ml}^{35}$. These levels are considerably higher than the usual undetectable levels in human blood $(\approx 1 \mathrm{pg} / \mathrm{ml})^{36}$. This mouse model was precisely chosen to sustain and expand NK cells, since a loss of NK cell function was deemed the underpinning reason why SMG evokes impaired anti-tumor responses of human lymphocytes. NK cell levels in blood fell in the later weeks when the differences in BLI between the groups reached discernible levels. It remains unclear if NK cells became exhausted in the later weeks (due to sustained IL-15 stimulation) or if they simply traffic away from the peripheral blood and are more abundant in the tissues and areas of sustained tumor growth.

Hematological tumor lysis in a 4-hour in vitro killing assay using mixed PBMCs is majorly attributed to the anti-tumor activity of NK cells; however, the ability to control tumor growth in vivo over a 6-week period might be a composite effect of all effector lymphocytes. While other effector lymphocytes like $\mathrm{CD} 8+\mathrm{T}$ cells could have played a role, we deem this unlikely as GvHD scores (a manifestation of the human $\mathrm{CD} 3+a \beta$ T-cells attacking xenogeneic tissue in the mouse) were not different between the SMG and $1 \mathrm{G}$ exposed PBMC conditions indicating that SMG did not affect the alloreactivity of CD8 $+\mathrm{a} \beta \mathrm{T}$-cells. Past evidence shows in vitro T-cell activity is suppressed after exposure to SMG ${ }^{37}$. However, in our in vivo model, there might be a recovery of the lost function over the duration of the experiment, particularly because the immunocompromised mice we used have a human IL-15 knock in that could have restored any detriments in a $\mathrm{T}$ T-cell function due to SMG. Since clinical symptoms of GvHD did not manifest until 3-4 weeks into the experiment, this might reflect similar GVHD onset patterns between the groups. While similar recovery is possible in NK cells too, failure to control tumor growth in the initial weeks might be contributing to tumor burden reaching an uncontrollable level before NK cells regain their activity. The more efficient 1G-exposed PBMCs could be more adept at killing K562 tumor cells in vivo during the initial weeks, which might have resulted in stunted tumor growth in the later weeks.

There is a critical need to identify effective countermeasures that can mitigate immune dysregulation during long duration spaceflight. Subcutaneous IL-2 injections are commonly used as an immunotherapeutic strategy to enhance graft-versus-leukemia effects in bone marrow transplant recipients by accelerating NK-cell reconstitution ${ }^{38}$. Additionally, zoledronic acid, an aminobisphosphonate that is used to prevent osteoporosis ${ }^{21}$ and currently being considered as a countermeasure to prevent decreases in bone mineral density due to weightlessness ${ }^{39}$, has also been shown to expand $y \delta-T$ cells in vivo and increase their anti-tumor activity in graft recipients ${ }^{40}$. We show here for the first time that that systemic administration of ZOL + IL2 'rescued' the anti-leukemia activity of SMG-exposed PBMCs in vivo. Indeed, while SMG exposed PBMCs were rendered completely ineffective against K562 leukemic growth in vivo, the tumor growth kinetics in mice that received SMG exposed PBMCs were identical to those that received $1 \mathrm{G}$ exposed PBMCs when ZOL + IL-2 was administered. This indicates that ZOL + IL-2 has the potential to completely reverse the negative impact of SMG on the antileukemic activity of human lymphocytes. It should be noted, however, that all PBMCs were returned to the $1 \mathrm{G}$ environment after adoptive transfer and we do not currently know if ZOL + IL-2 is capable of maintaining anti-leukemic activity during periods of microgravity. Nevertheless, these results indicate that 
$\mathrm{ZOL}+\mathrm{IL}-2$ could at least be an effective strategy to restore human lymphocyte function in conditions of $1 \mathrm{~g}$ (e.g. on return to Earth after a mission) or partial (e.g. on arrival at the Martian surface) gravity following prolonged periods of weightlessness.

\section{Conclusion}

In summary, we have demonstrated that exposure to SMG impairs the anti-leukemia activity of human effector immune cells in vivo as well as in vitro, and that ZOL+IL2 therapy improved the anti-leukemia activity of human effector immune cells after exposure to SMG. Limitations of the present study include the short-term exposure to microgravity, not identifying the lymphocyte subtypes affected by SMG, and the fact that all functional experiments were conducted in 1G. We conclude that microgravity plays a contributory role in the loss of tumor control and should be considered a risk factor for impaired antitumor immune responses during prolonged space voyages. Immuno-stimulating agents like ZOL+IL-2 may help mitigate clinical risks associated with immune dysregulation during prolonged spaceflight and its effectiveness as an immune system countermeasure in humans warrants investigation.

\section{Abbreviations}

SMG, simulated microgravity; PBMC, peripheral blood mononuclear cells; NK cell, Natural Killer cell; MHC, major histocompatibility complex; GVHD, graft-versus-host-disease; ZOL, zoledronic acid; IL-2, interleukin2; IL-15, interleukin-15; TNFa, tumor necrosis factor- alpha; IFNy, interferon-gamma; BLI, bioluminescent intensity.

\section{Declarations}

\section{Ethics approval and consent to participate:}

All study procedures were approved by the Institutional Review Board (IRB) at University of Arizona. All mouse experiments were done in compliance with Institutional Animal Care and Use Committee (IACUC) guidelines at the University of Arizona under an approved protocol.

\section{Consent for publication}

Not applicable

\section{Availability of data and materials}

The datasets used and/or analyzed during the current study are available from the corresponding author on reasonable request.

\section{Competing interests}

The authors declare that they have no competing interests. 


\section{Funding}

These studies were supported by NASA grants NNX16AB29G to RJS and 80NSSC19K1059 to RJS, EK and PLM.

\section{Authors' contributions}

RJS, PLM and EK designed the research. PLM, FLB, DMD and GMN performed the research. PLM, RJS, EK, MMM and BEC analyzed and/or interpreted the data. PLM and RJS wrote the manuscript. All coauthors edited the manuscript and approved its submission.

\section{Acknowledgements}

The authors thank Emely Hoffman and Jessica Stokes for assistance with the animal work.

\section{References}

1. Crucian B, Stowe R, Mehta $S$, et al. Immune system dysregulation occurs during short duration spaceflight on board the space shuttle. J Clin Immunol. 2013;33(2):456-465. doi:10.1007/s10875012-9824-7

2. Crucian B, Stowe R, Quiriarte H, Pierson D, Sams C. Monocyte phenotype and cytokine production profiles are dysregulated by short-duration spaceflight. Aviat Space Environ Med. 2011;82(9):857862. doi:10.3357/asem.3047.2011

3. Crucian BE, Stowe RP, Pierson DL, Sams CF. Immune system dysregulation following short- vs longduration spaceflight. Aviat Sp Environ Med. 2008;79(9):835-843. doi:10.3357/ASEM.2276.2008

4. Mehta SK, Laudenslager ML, Stowe RP, Crucian BE, Sams CF, Pierson DL. Multiple latent viruses reactivate in astronauts during Space Shuttle missions. Brain Behav Immun. 2014;41(1):210-217. doi:10.1016/j.bbi.2014.05.014

5. Crucian B, Stowe RP, Mehta S, Quiriarte H, Pierson D, Sams C. Alterations in adaptive immunity persist during long-duration space fl ight. 2015;(January):1-10. doi:10.1038/npjmgrav.2015.13

6. Mehta SK, Laudenslager ML, Stowe RP, et al. Latent virus reactivation in astronauts on the international space station. npj Microgravity. 2017;3(1):1-7. doi:10.1038/s41526-017-0015-y

7. Rooney B V, Crucian BE, Pierson DL, et al. Herpes Virus Reactivation in Astronauts During Spaceflight and Its Application on Earth. 2019;10(February):1-9. doi:10.3389/fmicb.2019.00016

8. Bigley $A B$, Agha NH, Baker FL, et al. NK cell function is impaired during long-duration spaceflight. Published online 2019:842-853. doi:10.1152/japplphysiol.00761.2018

9. Mylabathula PL, Li L, Bigley $A B$, et al. Simulated microgravity disarms human NK-cells and inhibits anti-tumor cytotoxicity in vitro. Acta Astronaut. 2020;174(March):32-40. doi:10.1016/j.actaastro.2020.03.023 
10. Bosma GC, Custer RP, Bosma MJ. A severe combined immunodeficiency mutation in the mouse. Nature. 1983;301(5900):527-530. doi:10.1038/301527a0

11. Shultz LD, Lyons BL, Burzenski LM, et al. Human Lymphoid and Myeloid Cell Development in NOD/LtSz-scid IL2R null Mice Engrafted with Mobilized Human Hemopoietic Stem Cells. J Immunol. 2005;174(10):6477-6489. doi:10.4049/jimmunol.174.10.6477

12. Cao X, Shores EW, Hu-Li J, et al. Defective lymphoid development in mice lacking expression of the common cytokine receptor $y$ chain. Immunity. 1995;2(3):223-238. doi:10.1016/10747613(95)90047-0

13. Shultz LD, Ishikawa F, Greiner DL. Humanized mice in translational biomedical research. Nat Rev Immunol. 2007;7(2):118-130. doi:10.1038/nri2017

14. Cooper MA, Bush JE, Fehniger TA, et al. In vivo evidence for a dependence on interleukin 15 for survival of natural killer cells. Blood. 2002;100(10):3633-3638. doi:10.1182/blood-2001-12-0293

15. Crucian BE, Choukèr A, Simpson RJ, et al. Immune system dysregulation during spaceflight: Potential countermeasures for deep space exploration missions. Front Immunol. 2018;9(JUN):1-21. doi:10.3389/fimmu.2018.01437

16. Cheever MA, Greenberg PD, Fefer A, Gillis S. Augmentation of the anti-tumor therapeutic efficacy of long-term cultured $\mathrm{T}$ lymphocytes by in vivo administration of purified interleukin 2. J Exp Med. 1982;155(4):968-980. doi:10.1084/jem.155.4.968

17. Mazumder A, Rosenberg SA. Successful immunotherapy of natural killer-resistant established pulmonary melanoma metastases by the intravenous adoptive transfer of syngeneic lymphocytes activated in vitro by interleukin 2. J Exp Med. 1984;159(2):495-507. doi:10.1084/jem.159.2.495

18. Rosenberg SA. IL-2: the first effective immunotherapy for human cancer. J Immunol. 2014;192(12):5451-5458. doi:10.4049/jimmunol.1490019

19. Muntasell A, Ochoa MC, Cordeiro L, et al. Targeting NK-cell checkpoints for cancer immunotherapy. Curr Opin Immunol. 2017;45:73-81. doi:10.1016/j.coi.2017.01.003

20. Kimpo MS, Oh B, Lee S. The Role of Natural Killer Cells as a Platform for Immunotherapy in Pediatric Cancers. Curr Oncol Rep. 2019;21(10):93. doi:10.1007/s11912-019-0837-8

21. Fobelo Lozano MJ, Sanchez-Fidalgo S. Adherence and preference of intravenous zoledronic acid for osteoporosis versus other bisphosphonates. Eur J Hosp Pharm Sci Pract. 2019;26(1):4-9. doi:10.1136/ejhpharm-2017-001258

22. Mei M, Xiang Z, Yang J, Xiang R. Efficacy of zoledronic acid for prevention of bone loss in early-stage breast cancer patients receiving adjuvant therapy: A meta-analysis of 13 randomized controlled trials. Curr Probl Cancer. Published online November 2019:100507. doi:10.1016/j.currproblcancer.2019.100507

23. Liu H, Wang S-H, Chen S-C, Chen C-Y, Lo J-L, Lin T-M. Immune modulation of CD4(+)CD25(+) regulatory T cells by zoledronic acid. BMC Immunol. 2016;17(1):45. doi:10.1186/s12865-016-0183-7

24. Salaroglio IC, Campia I, Kopecka J, et al. Zoledronic acid overcomes chemoresistance and immunosuppression of malignant mesothelioma. Oncotarget. 2015;6(2):1128-1142. 
doi:10.18632/oncotarget.2731

25. Monkkonen H, Ottewell PD, Kuokkanen J, Monkkonen J, Auriola S, Holen I. Zoledronic acid-induced IPP/Apppl production in vivo. Life Sci. 2007;81(13):1066-1070. doi:10.1016/j.Ifs.2007.08.007

26. Caccamo N, Meraviglia S, Scarpa F, et al. Aminobisphosphonate-activated gammadelta T cells in immunotherapy of cancer: doubts no more. Expert Opin Biol Ther. 2008;8(7):875-883. doi:10.1517/14712598.8.7.875

27. Hamilton E, Clay TM, Blackwell KL. New perspectives on zoledronic acid in breast cancer: potential augmentation of anticancer immune response. Cancer Invest. 2011;29(8):533-541. doi:10.3109/07357907.2011.605413

28. Lai HY, Chou TY, Tzeng CH, Lee OKS. Cytokine profiles in various graft-versus-host disease target organs following hematopoietic stem cell transplantation. Cell Transplant. 2012;21(9):2033-2045. doi:10.3727/096368912X653110

29. Naserian S, Leclerc M, Thiolat A, et al. Simple, reproducible, and efficient clinical grading system for murine models of acute graft-versus-host disease. Front Immunol. 2018;9(JAN):1-9. doi:10.3389/fimmu.2018.00010

30. Paley MA, Prescher JA. Bioluminescence: a versatile technique for imaging cellular and molecular features. Medchemcomm. 2014;5(3):255-267. doi:10.1039/C3MD00288H

31. Duda J, Karimi M, Negrin RS, Contag CH. Methods for imaging cell fates in hematopoiesis. Methods Mol Med. 2007;134:17-34. doi:10.1007/978-1-59745-223-6_2

32. Stokes J, Hoffman EA, Zeng Y, Larmonier N, Katsanis E. Post-transplant bendamustine reduces GvHD while preserving GvL in experimental haploidentical bone marrow transplantation. $\mathrm{Br} \mathrm{J}$ Haematol. Published online 2016. doi:10.1111/bjh.14034

33. JP F, J H, M Y, K G, J A. Y $\delta \mathrm{T}$ cells for cancer immunotherapy: A systematic review of clinical trials. Oncoimmunology. 2014;3(1). doi:10.4161/ONCI.27572

34. Mylabathula PL, Li L, Bigley AB, et al. Simulated microgravity disarms human NK-cells and inhibits anti-tumor cytotoxicity in vitro. Acta Astronaut. 2020;174. doi:10.1016/j.actaastro.2020.03.023

35. 030890 - NOD.Cg-Prkdc < scid > II2rg < tm1Wjl > Tg(IL15)1Sz/SzJ. Accessed June 23, 2020. https://www.jax.org/strain/030890

36. Bergamaschi C, Bear J, Rosati M, et al. Circulating IL-15 exists as heterodimeric complex with soluble IL-15Ra in human and mouse serum. Blood. 2012;120(1):e1-e8. doi:10.1182/blood-2011-10-384362

37. Hauschild S, Tauber S, Lauber B, Thiel CS, Layer LE, Ullrich O. T cell regulation in microgravity - The current knowledge from in vitro experiments conducted in space, parabolic flights and ground-based facilities. Acta Astronaut. 2014;104(1):365-377. doi:10.1016/j.actaastro.2014.05.019

38. Miller JS, Soignier Y, Panoskaltsis-Mortari A, et al. Successful adoptive transfer and in vivo expansion of human haploidentical NK cells in patients with cancer. Blood. 2005;105(8):3051-3057. doi:10.1182/BLOOD-2004-07-2974 
39. Cavanagh PR, Licata AA, Rice AJ. Exercise and pharmacological countermeasures for bone loss during long-duration space flight. Gravit Space Biol Bull. 2005;18(2):39-58.

40. A B, A Z, A P, et al. Zoledronic acid boosts $\gamma \delta$ T-cell activity in children receiving $a \beta+T$ and CD19 + cell-depleted grafts from an HLA-haplo-identical donor. Oncoimmunology. 2016;6(2). doi:10.1080/2162402X.2016.1216291

\section{Figures}

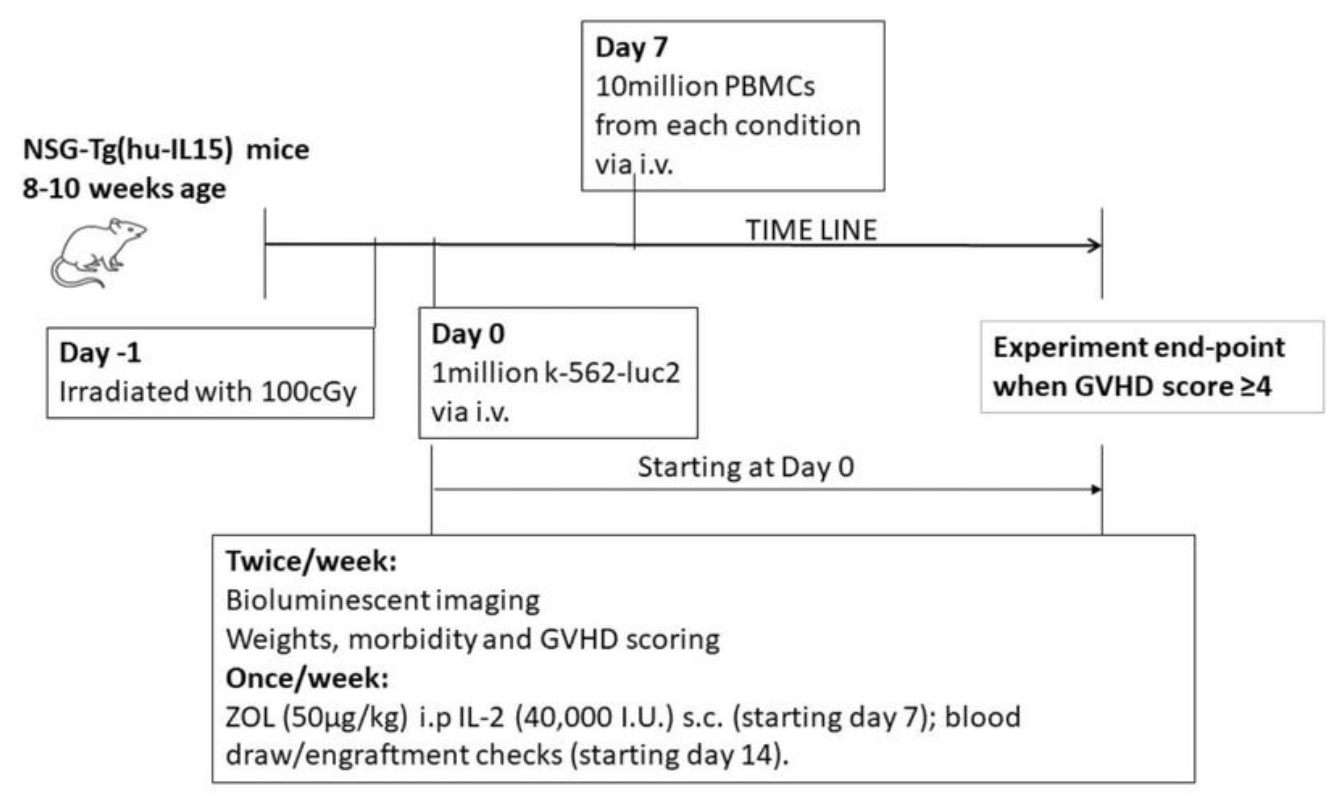

\section{Figure 1.}

\section{Figure 1}

Experimental design 


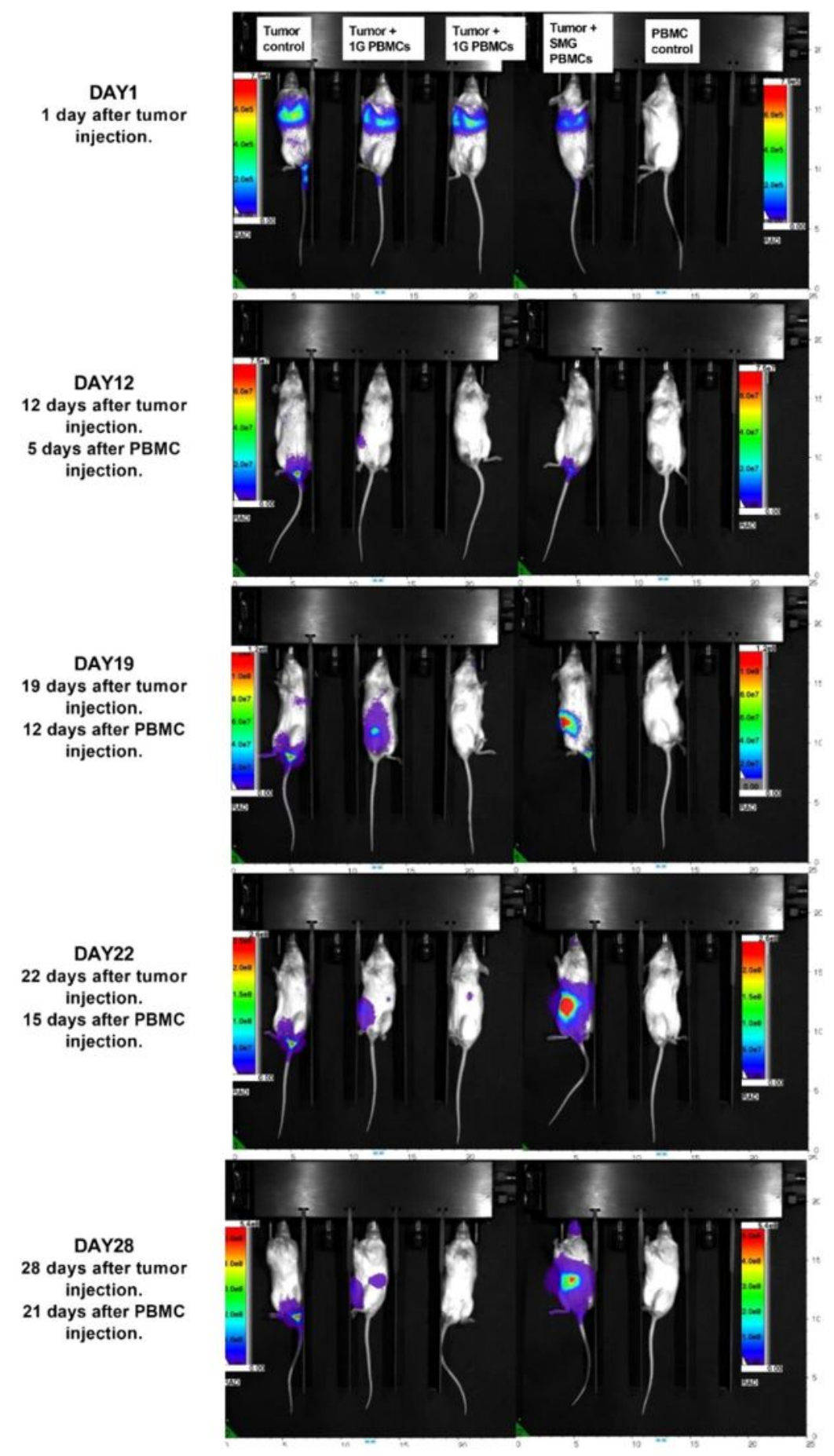

Figure 2.

\section{Figure 2}

Bioluminescent intensity (BLI) images. Days 1, 12, 19, 22 and 28 of the experiment. Lane 1: tumor control, lanes 3, 5: tumor+ 1G PBMCs, lane 6: tumor+ SMG PBMCs, lane 8: PBMC control. 
3a.

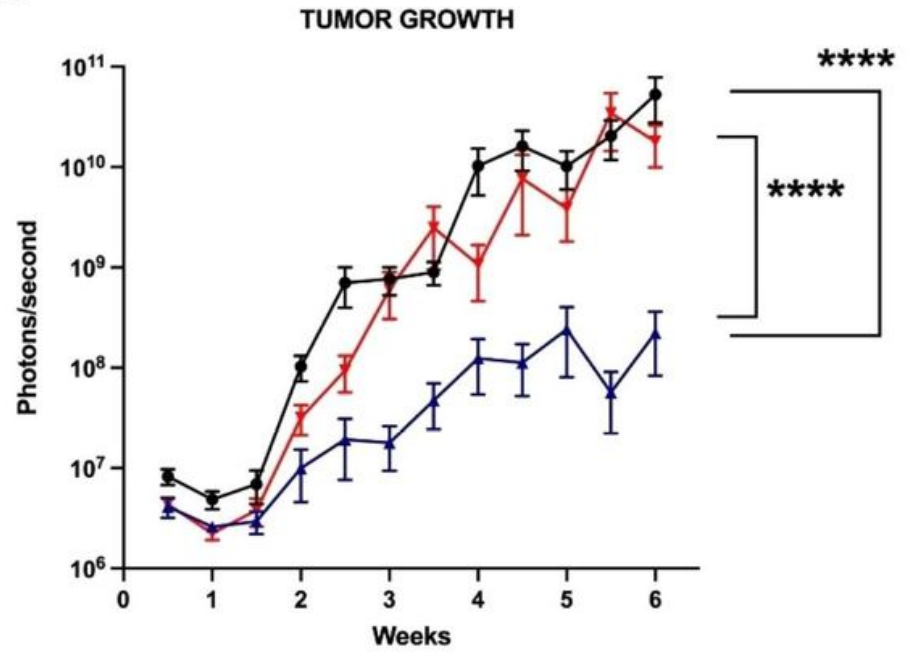

$3 \mathrm{c}$

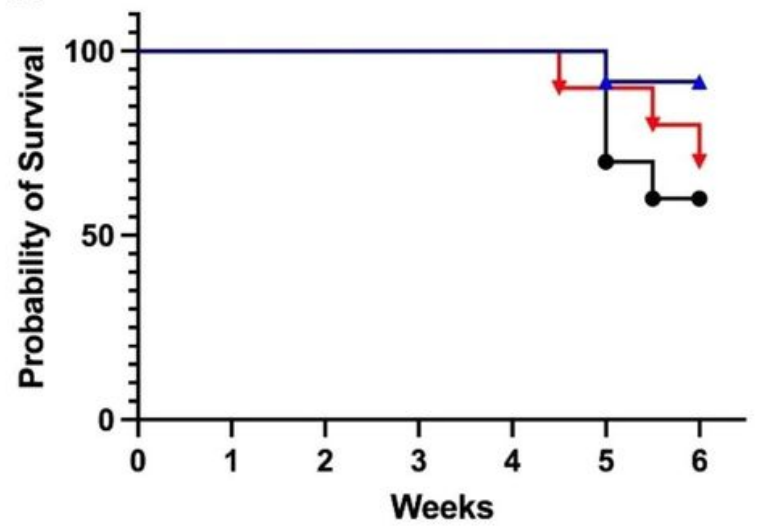

3b.

PEAK BLI

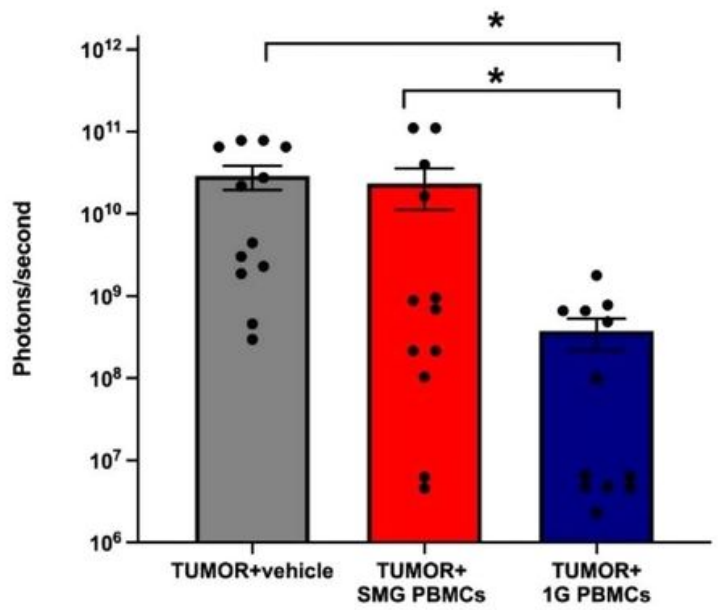

3d.

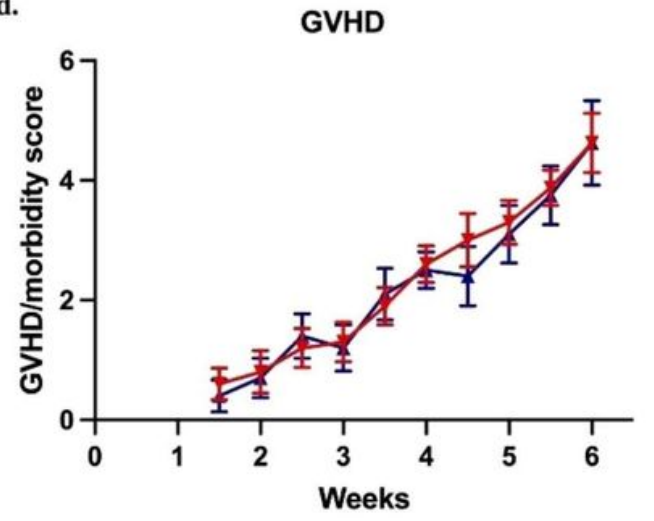

Figure 3.

Figure 3

Effect of SMG on effector immune cell function in vivo. Exposure to SMG impaired effector immune cell ability to control tumor growth in an in vivo model (3a.) $(* \star \star *=p<0.0001)$. Exposure to SMG also significantly increased peak BLI score during the experiment (3b.) ( ${ }^{*}$ denotes $p<0.05$, $* \star$ denotes $p<0.01$ ). Exposure to SMG did not affect survival (3c.) and GVHD incidence (3d.). $N=12, M E A N \pm S E M$. 
4a.

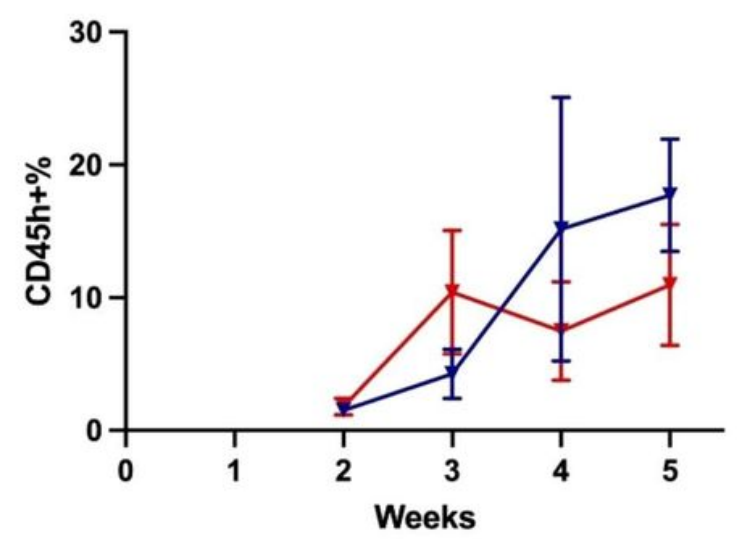

4c.

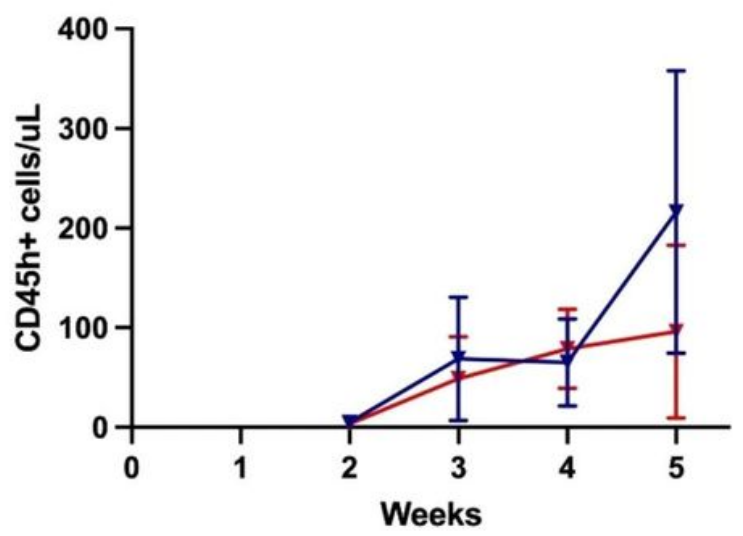

$4 b$.

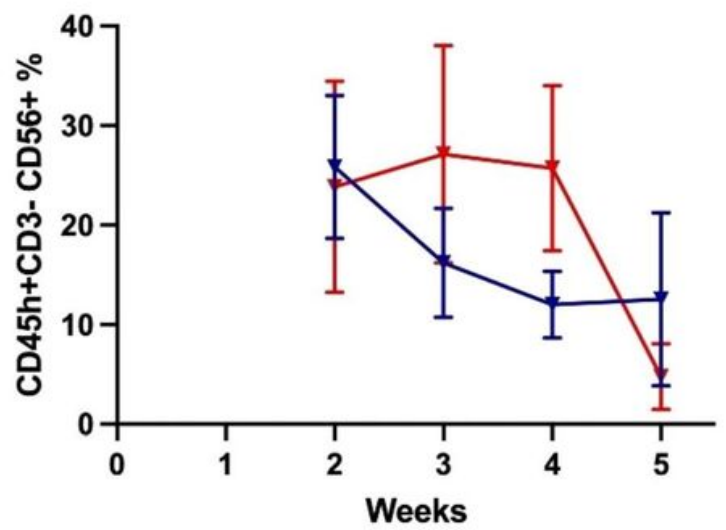

4d.

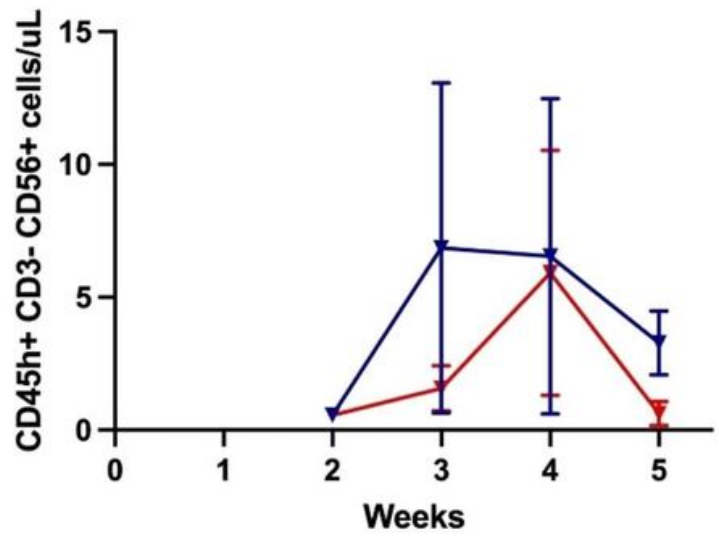

Figure 4.

Figure 4

Effect of exposure to SMG on human cell engraftment dynamics in vivo in the absence of tumor.

Exposure to SMG did not impair human PBMCs' (4a., 4c.) and NK cells' (4b., 4d.) ability to engraft in the presence of tumor. $\mathrm{N}=10, \mathrm{MEAN} \pm$ SEM. 
5a.

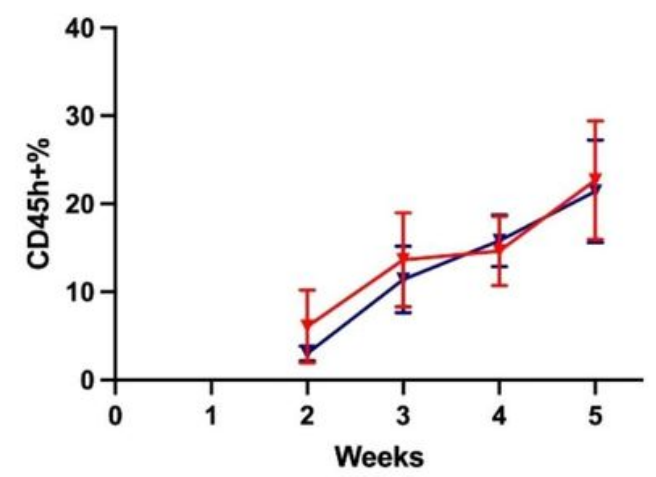

5c.

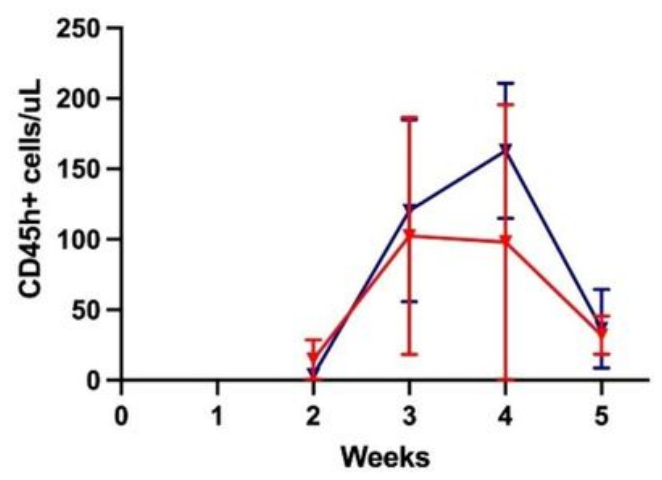

5b.

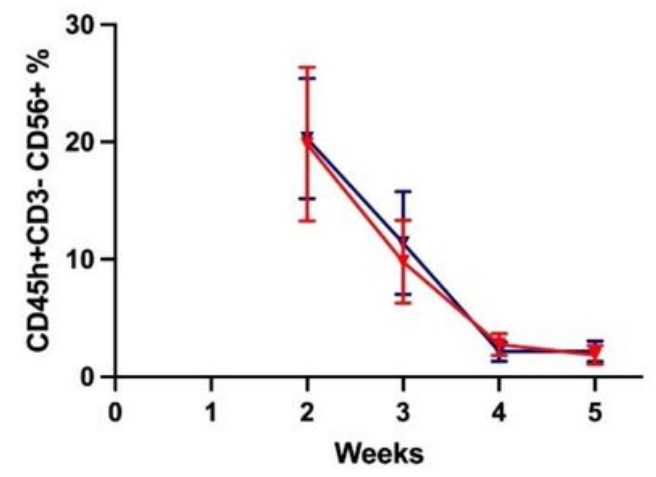

5d.

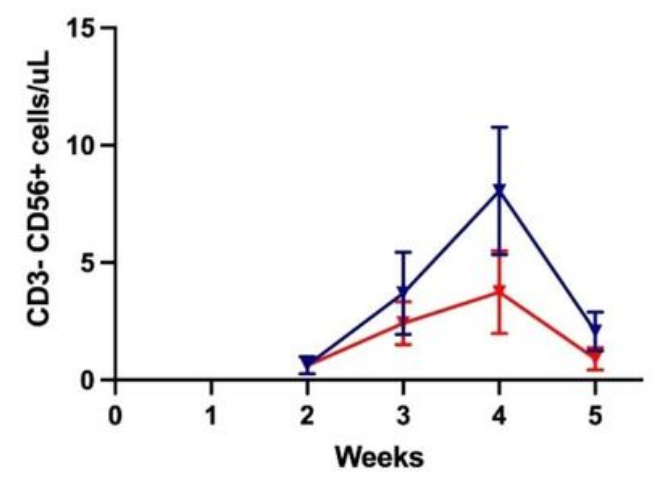

Figure 5.

\section{Figure 5}

Effect of SMG on human cells engraftment in vivo in the presence of tumor. Exposure to SMG did not impair human PBMCs' (5a., 5c.) and NK cells' (5b., 5d.) ability to thrive in vivo. $\mathrm{N}=5, \mathrm{MEAN} \pm$ SEM. 
$6 a$.

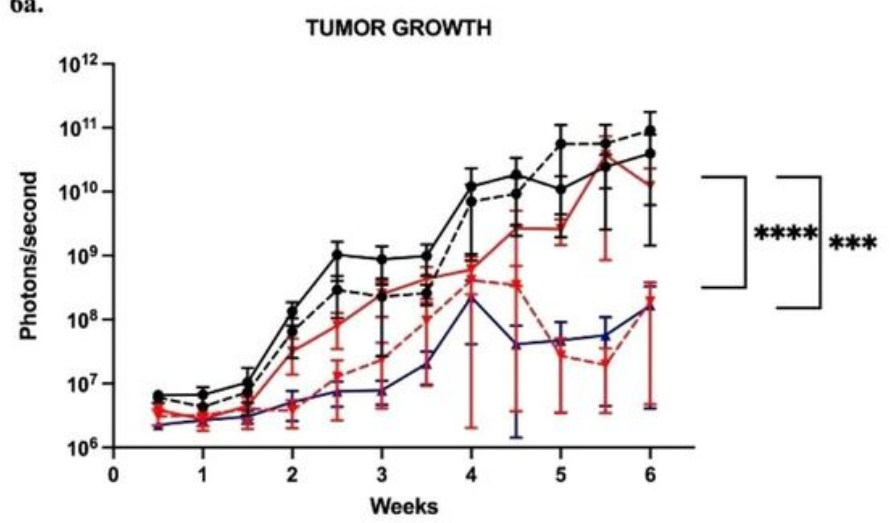

$6 c$.

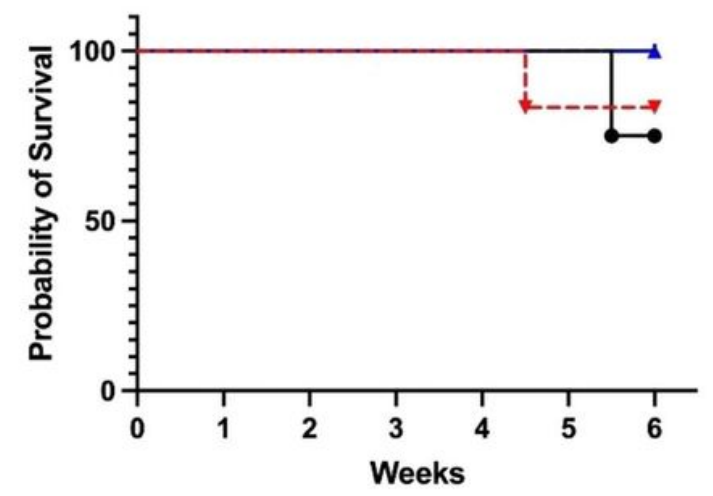

6b.
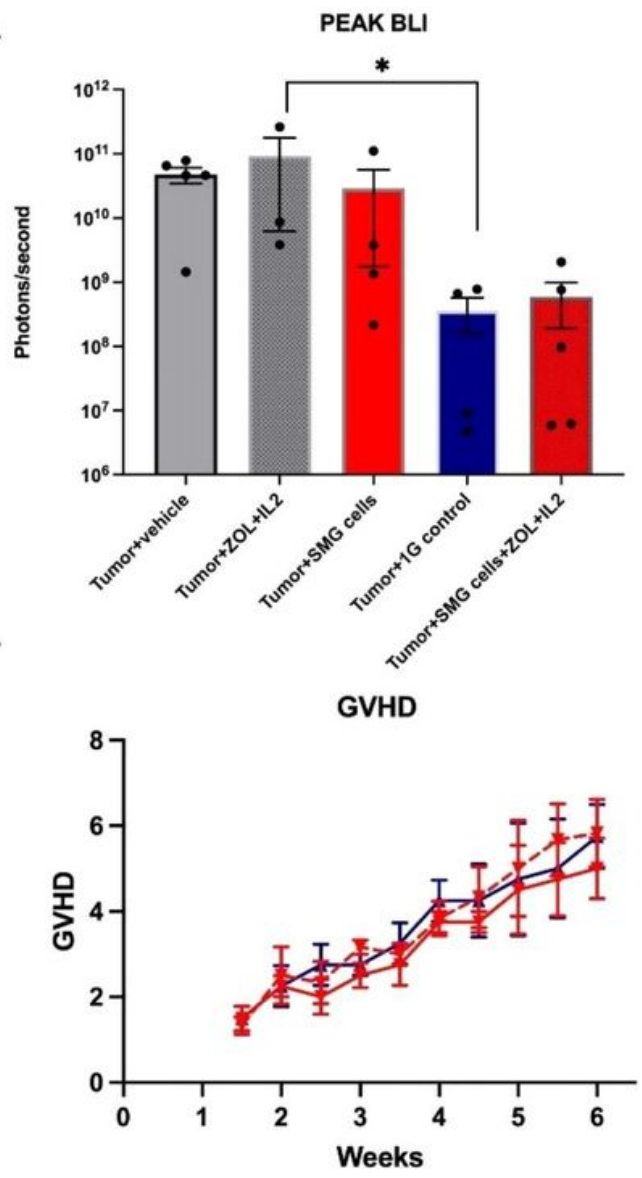

Figure 6.

Figure 6

Effect of zoledronic acid+IL2 (ZOL+IL2) therapy on SMG induced suppression of in vivo anti-leukemia activity. ZOL+IL2 therapy abrogated SMG induced loss of anti-leukemia activity in vivo (6a.), did not affect peak BLI (6b.), survival (6c.), GVHD incidence (6d.). N=5, MEAN $\pm S E M$. 
$7 a$.

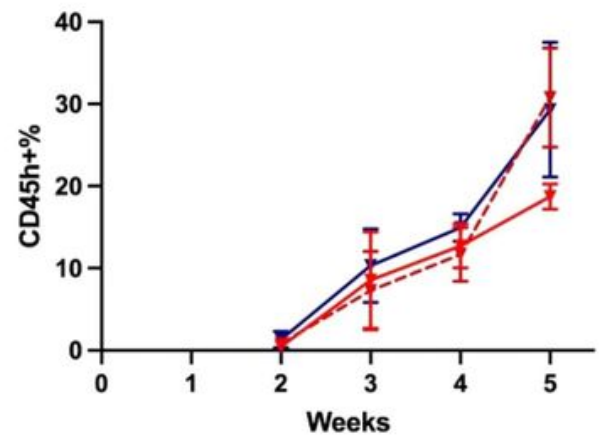

7c.

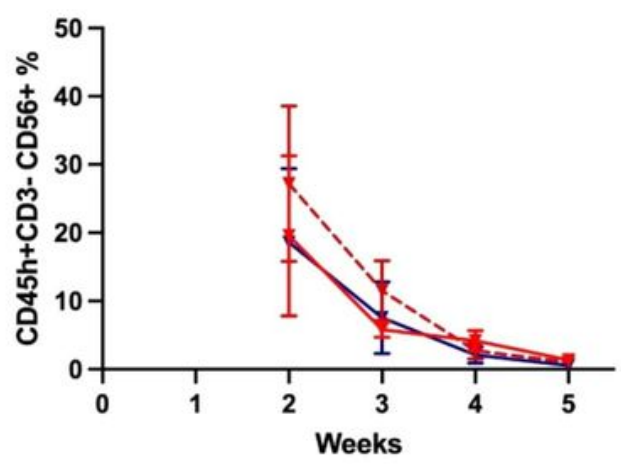

7 e.

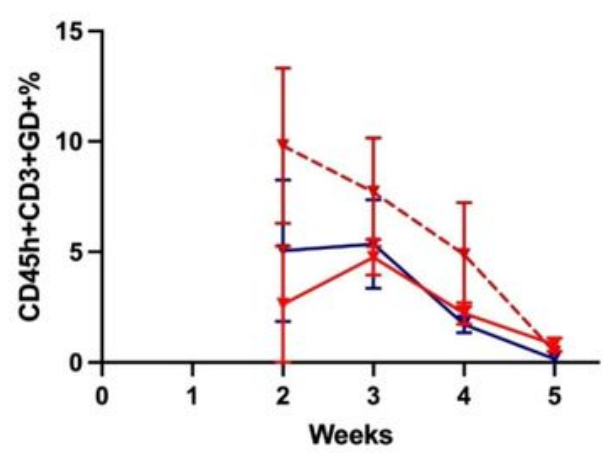

$7 b$.

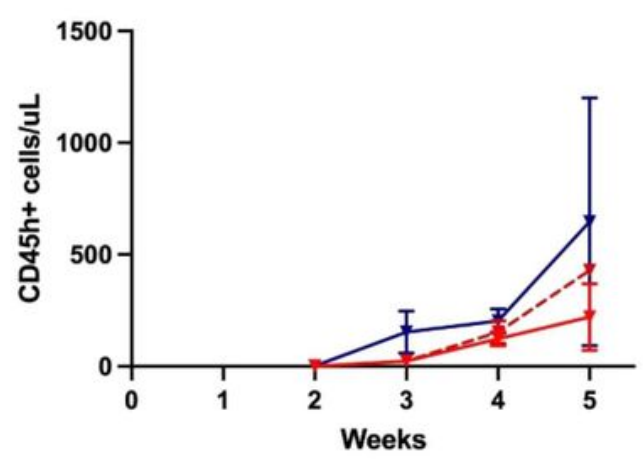

7d.

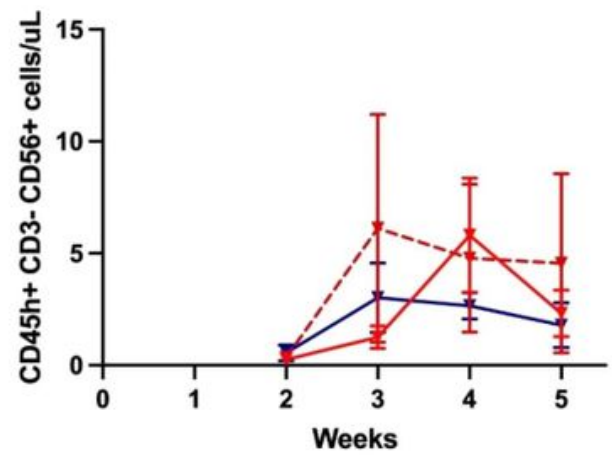

$7 f$.

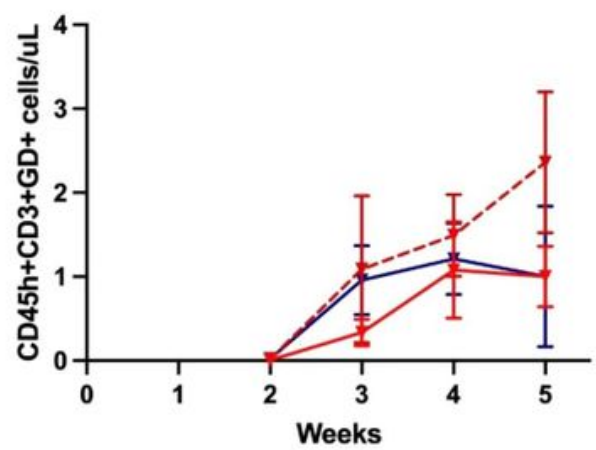

Figure 7.

\section{Figure 7}

Effect of SMG and ZOL+IL2 therapy on human cells engraftment in vivo in the presence of tumor. ZOL+IL2 therapy did not alter human PBMCs' (7a., 7b.) and NK cells' (7c., 7d.) and ү反-T cell (7e., 7f.) engraftment dynamics. $\mathrm{N}=5 \mathrm{MEAN} \pm$ SEM. 